\title{
Gastric Drip Feeding in Patients with Glycogen Storage Disease Type I: Its Effects on Growth and Plasma Lipids and Apolipoproteins
}

\author{
J. FERNANDES, P. ALAUPOVIC, AND J. M. WIT \\ Department of Pediatrics [J.F.], University Hospital, 59 Oostersingel, 9713 EZ Groningen and Wilhelmina \\ Children's Hospital [J.M.W.], University of Utrecht, 137 Nieuwe Gracht, 3512 LK Utrecht, The Netherlands, and \\ Lipoprotein and Atherosclerosis Research Program [P.A.J Oklahoma Medical Research Foundation, \\ Oklahoma City, OK 73104
}

\begin{abstract}
In 19 patients with a deficiency of glucose6-phosphatase and 1 patient with a deficiency of glucose6-phosphate translocase, the effect of nocturnal gastric drip feeding (GDF) on growth and plasma lipids and apolipoproteins was studied. The effect on growth was estimated by determining the height standard deviation score (SDS) of the patients and comparing its changes ( $\triangle$ SDS) over 4-, 2-, and 1-y periods before and 1-, 2-, 5-, and 8-y periods after the institution of GDF. The effect of GDF on plasma lipids and apolipoproteins was investigated by following the concentrations of triglycerides, cholesterol, and apolipoproteins A-I, A-II, B, C-I, C-II, C-III, and E. Growth caught up significantly or remained in the normal range in 14 patients. They were defined as responders to GDF. In the other six patients, growth caught up insufficiently or showed a further deceleration. They were defined as nonresponders to GDF. GDF had only a temporary and marginal effect on plasma lipids and apolipoproteins, but after 5-8 y, the levels of plasma triglycerides, cholesterol, apolipoprotein, B, C-I, C-II, C-III, and E increased further in both responders and nonresponders, whereas apolipoproteins A-I and A-II decreased in nonresponders. There were minor differences in the levels of lipids and apolipoproteins between responders and nonresponders without any discernible trends during the first years of GDF. After 5-8 y, the levels of cholesterol, triglycerides and apolipoproteins B, C-I, C-II, C-III, and E seemed to be higher, and apolipoproteins A-I and A-II lower, in nonresponders than in responders, but the differences were not significant. (Pediatr Res 25:327-331, 1989)
\end{abstract}

\section{Abbreviations}

GSD-IA, glycogen storage disease caused by deficiency of glucose-6-phosphatase

GSD-IB, glycogen storage disease caused by deficiency of glucose-6-phosphate translocase

GDF, gastric drip feeding

height SDS, height standard deviation score

The administration of nocturnal GDF to patients with GSDIA or GSD-IB has markedly improved their growth $(1,2)$. With GDF such main metabolic abnormalities as hyperlactacidemia,

Reccived June 9. 1988; accepted October 27, 1988

Correspondence to J. Fernandes, Department of Pediatrics, University Hospital, 59 Oostersingel, 9713 EZ Groningen. The Netherlands. hyperuricemia, and hyperlipidemia also improve initially, but the long-term effects vary (3).

We treated 20 patients (19 with GSD-IA and 1 with GSD-IB) with GDF during 2 to $12 \mathrm{y}$, and the majority showed catch-up growth. However, some patients continued to grow at or below the lowest percentiles of the height references or lagged further behind. The height SDS was found to correlate inversely with the urinary excretion of some important metabolites of glycogenolysis (lactate) and the citric acid cycle (2-oxoglutarate) (4). Assuming that a similar relation might exist between growth and other metabolic abnormalities, we studied the concentrations of plasma lipids and apolipoproteins before and during GDF. Hyperlipidemia and hyperlipoproteinemia are well-known abnormalities in GSD-I $(2,5)$, and the plasma apolipoprotein profile shows a characteristic pattern (6). It is not known whether the abnormal apolipoprotein profile persists during GDF irrespective of the condition of the patient or improves in concert with an improved growth. Therefore, we studied this issue in $20 \mathrm{GSD}-\mathrm{I}$ patients with a large range of ages and height SDS from the beginning of GDF until adolescence.

\section{PATIENTS}

The study included 20 patients, aged 0.03 to $8.51 \mathrm{y}$ at the institution of GDF. Glucose-6-phosphatase deficiency was diagnosed in 19 patients by enzyme assay of a liver biopsy, except for one patient who is a brother of a biopsy-documented case. Glucose-6-phosphate translocase deficiency was diagnosed in the liver biopsy of one patient (case 9). The patients were free from other diseases, but had the usual abnormalities due to their primary disorder, such as marked hepatomegaly, tendency to hypoglycemia, hyperlactacidemia, hyperuricemia, hyperlipidemia, and hyperlipoproteinemia. The diet before the institution of GDF consisted of frequent meals around the clock. The average composition of the diet was: carbohydrates, 60-70 energy\% (lactose- and sucrose-restricted), protein 10-15 energy \%, fat $20-25$ energy $\%$ with a polyunsaturated/saturated $(\mathrm{P} / \mathrm{S})$ fatty acid ratio of 1:3. After the institution of GDF, the amount of carbohydrates decreased slightly to 60-65 energy $\%$ with more emphasis on starch. The amount of fat increased reciprocally, and the $\mathrm{P} / \mathrm{S}$ ratio increased to $1: 1$. The GDF was a low-lactose adapted cow's milk formula with extra maltodextrin, amounting to $4-8 \mathrm{mg}$ glucose $\cdot \mathrm{kg}^{-1} \cdot \mathrm{min}^{-1}$, administered during $10-12 \mathrm{~h} /$ night. The GDF provided approximately $35 \%$ of the total energy intake/d.

\section{MATERIALS AND METHODS}

Growth analysis. Data on statural growth before the start of GDF were collected from the referring pediatricians and from 
our own clinic. Our measurements of height were performed on a Harpenden stadiometer. All heights were expressed as SDS, according to Tanner et al. (7). The SDS is defined as the patient's height minus the mean for the actual age, divided by the SD at that age. For this purpose, the 1980 nation-wide Dutch references (8) were used. In addition, the SDS corrected for parental height was calculated by subtracting the mean parental SDS from the child's SDS value. The parental SDS was calculated using the mean and SD of adult men's and women's heights in the 1965 nation-wide Dutch references (9). Normally, the height SDS of a child remains stable after the age of approximately $4 \mathrm{y}$, which signifies that heights tend to keep their track on the percentile charts. Deviation from the normal track, i.e. a change in height SDS, is the growth parameter of interest when the effect of a disease or of a possibly growth-promoting treatment is investigated. We therefore calculated the change of height SDS over periods of 1,2, and 4 y before and 1,2, 5, and 8 y during GDF treatment. These values were all estimated by interpolation of the available height measurements. The values obtained over 1 , 2,4 , and 5-y periods were compared with the $95 \%$ confidence intervals in a Finnish healthy child population (10). Wt-forheight was expressed as a percentage of the median wt-for-height from the recent Dutch growth study (8).

Laboratory methods. Venipuncture was performed for routine assay of electrolytes, urate, and liver and kidney function. Additional blood was taken for the lipoprotein studies with the consent of the parents and/or the patients. The venipuncture was performed in the nonfasting state because of the risk of fasting hypoglycemia. The preceding breakfast consisted of 1-2 slices of bread with margarine, jam, or marmalade, and tea with low-fat milk. EDTA blood samples were centrifuged at low speed, and the plasma was used for the determination of triglycerides and cholesterol by routine procedures $(11,12)$. The remaining plasma was shipped immediately by airmail at ambient temperature from Groningen to Oklahoma City, OK, for apolipoproteins analyses. Plasma apolipoproteins were measured by electroimmunoassays as described earlier (6).

Statistical methods. As a result of skewed distributions of lipid and apolipoprotein values in some patients, the significance of differences was estimated by the Wilcoxon rank sum test. The level of significance was taken as $p \leq 0.05$.

\section{RESULTS}

Growth analysis. The height SDS at the start of GDF and its changes before and after the institution of GDF are shown in Table 1. Before treatment, a negative growth deviation is seen in almost all children, and in many cases the height SDS is below the 2.5 percentile. After the institution of GDF, catch-up growth, indicated by positive SDS changes, is seen in most children. The SDS changes are above the $p=95$ value in many cases. When the data are analyzed on an individual basis, several patterns emerge. The most common pattern is growth retardation before the onset of treatment, followed by catch-up growth for a few years, and subsequently stable growth parallel to the percentile curves. This pattern is seen in cases $1-9$, except that case 2 already showed accelerated growth before the start of GDF, probably because of more frequent feeding. These cases showed a normal distribution of height SDS (corrected for parental height SDS) from -1.14 to +2.86 after 5 y of GDF. Two patients, cases 10 and 11 , had a normal growth before GDF, which did not substantially change subsequently,

Cases 1-11 all have a growth pattern that can be considered as a satisfactory response to GDF. Two patients, cases 12 and 13 , with significant catch-up growth can be expected to attain ultimately normal height SDS, although their height SDS at $5 \mathrm{y}$ still were subnormal.

Case 14 showed catch-up growth after the start of GDF, but the period of observation was not long enough to be certain that this catch-up growth will be adequate.

Cases 15 and 16 had an early growth acceleration, followed by a lag. After 5 y of GDF they, and case 17, still showed a low height SDS (corrected for parental height SDS). This may be considered an insufficient response.

Finally, three cases, $18-20$, had a continued deceleration of growth, resulting in lower height SDS values during GDF than

Table 1. Height SDS and its changes among 20 patients with GSD-IA and GSD-IB before and during GDF

\begin{tabular}{|c|c|c|c|c|c|c|c|c|c|c|c|c|}
\hline \multirow[b]{2}{*}{ Case } & \multirow[b]{2}{*}{ Initials } & \multicolumn{3}{|c|}{ At start of GDF } & \multirow{2}{*}{$\begin{array}{c}\begin{array}{c}\text { After } 5 \text { y } \\
\text { GDF }\end{array} \\
\text { Height } \\
\text { SDS (corr)* }\end{array}$} & \multicolumn{3}{|c|}{ Before GDF: $\triangle$ SDS over } & \multicolumn{4}{|c|}{ During GDF: $\triangle \mathrm{SDS}$ over } \\
\hline & & Age & $\begin{array}{l}\text { Height } \\
\text { (SDS) }\end{array}$ & $\begin{array}{l}\text { Height SDS } \\
\text { (corr) }\end{array}$ & & $4 y$ & $2 y$ & $1 \mathrm{yr}$ & $1 \mathrm{y}$ & $2 y$ & $5 \mathrm{y}$ & $8 y$ \\
\hline 1 & MB & 6.57 & -3.65 & -4.09 & -0.96 & $-2.38 \dagger$ & $-0.82 \dagger$ & -0.07 & $+1.70 \dagger$ & $+2.48 \dagger$ & $+3.13 \dagger$ & +3.26 \\
\hline 2 & DD & 7.52 & -1.45 & -1.85 & -0.57 & +0.89 & +0.38 & $+1.02 \dagger$ & $+0.98 \dagger$ & $+1.40^{\dagger}$ & $+1.28 \dagger$ & +2.38 \\
\hline 3 & RS & 5.30 & -4.22 & -2.87 & -0.97 & $-2.32 \dagger$ & $-0.93 \uparrow$ & $-0.79 \dagger$ & $+1.12 \dagger$ & $+1.89 \dagger$ & $+1.90 \dagger$ & +2.25 \\
\hline 4 & PS & 8.51 & -2.94 & -2.14 & -1.14 & -0.77 & -0.09 & -0.18 & $+0.56 \dagger$ & $+0.94 \dagger$ & $+1.00 \dagger$ & +1.71 \\
\hline 5 & TD & 5.18 & -2.49 & -0.79 & +2.16 & -0.80 & -0.20 & -0.34 & $+1.85 \dagger$ & $+2.21 \dagger$ & $+2.95 \dagger$ & \\
\hline 6 & MG & 3.68 & -0.98 & -2.12 & -0.67 & & -0.95 & -0.07 & +0.39 & -0.01 & $+1.45 \dagger$ & +1.13 \\
\hline 7 & ME & 1.59 & +0.42 & +0.32 & +2.86 & & & -0.35 & $+1.90 \dagger$ & $+2.11 \dagger$ & $+2.54 \dagger$ & +1.61 \\
\hline 8 & $\mathrm{LA}$ & 0.59 & -1.32 & -1.56 & +0.84 & & & -1.85 & & +1.64 & $+2.40 \dagger$ & \\
\hline 9 & RP & 4.57 & -3.43 & -2.19 & $+0.01 \S$ & & -0.18 & -0.19 & & $+1.40 \dagger$ & $+2.20+\S$ & \\
\hline 10 & DS & 4.77 & -1.11 & -0.31 & $-0.86^{\circ}$ & +0.26 & -0.28 & +0.32 & -0.29 & -0.08 & -0.55 & -0.75 \\
\hline 11 & ID & 4.69 & -1.89 & -1.17 & & +0.96 & +0.10 & -0.10 & +0.17 & -0.06 & & \\
\hline 12 & HM & 8.33 & -3.50 & -4.25 & -2.44 & $-1.55 \dagger$ & $-1.08 \dagger$ & -0.34 & +0.29 & $+0.60 \dagger$ & $+1.81 \dagger$ & \\
\hline 13 & MR & 5.19 & -4.03 & -4.51 & -2.63 & -1.32 & -0.03 & +0.22 & $+0.99 \dagger$ & $+0.98 \dagger$ & $+1.88 \dagger$ & \\
\hline 14 & $\mathrm{EO}$ & 0.20 & -1.53 & -2.39 & & & & & +0.82 & +0.59 & & \\
\hline 15 & $\mathrm{ER}$ & 4.81 & -4.85 & -2.68 & -2.41 & $-3.78 \dagger$ & -0.51 & -0.50 & $+1.47 \dagger$ & $+1.58 \dagger$ & +0.27 & -0.38 \\
\hline 16 & MD & 4.98 & -3.43 & -3.23 & -3.97 & $-3.58 \dagger$ & -0.69 & -0.29 & +0.34 & +0.52 & -0.74 & -1.62 \\
\hline 17 & $C G$ & 4.48 & -2.17 & -3.31 & -2.67 & -0.17 & -0.45 & -0.16 & +0.46 & +0.69 & +0.64 & \\
\hline 18 & SN & 5.95 & -0.91 & -1.20 & & -0.26 & 0.00 & +0.05 & -0.49 & $-1.04 t$ & & \\
\hline 19 & $\mathrm{AN}$ & 1.61 & -0.64 & -0.93 & -1.79 & & & +0.25 & -0.34 & -0.73 & -0.86 & \\
\hline 20 & NS & 0.03 & +0.55 & +1.34 & & & & & -0.57 & -1.80 & & \\
\hline
\end{tabular}

* Patient's height SDS corrected for parental height SDS.

$\dagger$ Height SDS changes exceeding the $95 \%$ confidence intervals.

* SDS over 6-7 mo.

$\$$ SDS over 4 y after start of GDF because data after 5 y were unavailable. 
at its start. The first two must be considered as total nonresponders. The last patient is still very young, and the decrease of her height SDS may be the normal crossing of percentiles seen in genetically short children in the first years of life.

The weight (mean \pm SD) of the patients was $107 \pm 7 \%$ of the median wt-for-height. Wt-for-height increased slightly but not significantly (113 $\pm 12 \%)$ after 4 y of GDF.

Plasma lipids and apolipoproteins. Plasma lipid and apolipoprotein concentrations of patients responding to the GDF treatment (cases 1-14, see "Discussion") are shown in Table 2. As previously demonstrated (6), the patients' lipid and apolipoprotein profiles were abnormal before initiation of GDF. Triglycerides and apolipoproteins B, C-I, C-II, C-III, and E were all increased, A-I decreased, in comparison with normolipidemic, asymptomatic children of similar age (6). After the beginning of treatment with GDF fluctuations and changes in the concentrations of plasma triglycerides, cholesterol and all apolipoproteins were observed. The changes never reached statistical significance at any of the time intervals. After 1-2 y of GDF, the concentrations of triglycerides and cholesterol tended to decrease slightly, but after prolonged treatment, both triglyceride and cholesterol values were higher than at the beginning of GDF treatment. Concentrations of apolipoproteins A-I and A-II remained unchanged throughout the entire treatment period. There was some initial decrease in the apolipoprotein B concentrations, but after 5-8 y of GDF the apolipoprotein B levels were as high or higher than before the treatment. The initially high levels of apolipoprotein $C$ peptides and apolipoprotein $E$ remained unchanged, or increased further, after 5-8 y of GDF.

Similar trends, but without any transient decrease of some apolipoproteins, were also observed in lipid and apolipoprotein profiles of patients not responding to the GDF treatment (cases 15-20, Table 3). However, the increase in the concentration of cholesterol after 5-8 y was statistically significant when compared to that at the beginning of GDF. The increase of triglyceride levels after prolonged treatment with GDF was not statistically significant because of the small number of samples available for analysis and the relatively wide range of values. The same argument applies to the markedly increased levels of apolipoproteins B, C-I, C-II, C-III, and E after 5-8 y of GDF. The concentrations of apolipoprotein A-I and apolipoprotein A-II decreased steadily.

A comparison of lipid and apolipoprotein profiles estimated at different time intervals between responders and nonresponders to GDF therapy shows that both groups of patients were characterized by abnormal concentrations of plasma triglycerides and all apolipoproteins with the possible exception of apolipoprotein A-II. Small differences in the levels of these constituents were statistically insignificant. During prolonged treatment with GDF, the elevated levels of triglycerides, cholesterol, and most apolipoproteins were maintained in both responders and nonresponders. However, from 1-2 y of treatment onward, the concentrations of triglycerides, cholesterol, apolipoproteins B, C-III, and E of nonresponders tended to be higher than those of responders, especially at the interval of 5-8 y, whereas apolipoprotein A-I levels behaved reversely. As already stated, the lack of statistical significance of the differences between responders and nonresponders at these later intervals is most probably due to the relatively small number of samples available for analysis and wide variations in the lipid and apolipoprotein values of individual patients. However, the mean values for lipids and apolipo-

Table 2. Plasma lipid and apolipoprotein concentrations (in $\mathrm{mg} / \mathrm{dL}$ ) (mean $\pm S D$ ) in patients with GSD-IA and GSD-IB responding to GDF treatment (cases 1-14) and in control subjects (Ref. 6)

\begin{tabular}{|c|c|c|c|c|c|c|c|c|c|}
\hline \multirow[b]{2}{*}{ Treatment } & \multicolumn{2}{|c|}{ Lipids } & \multicolumn{7}{|c|}{ Apolipoproteins } \\
\hline & $\mathrm{TG}^{*}$ & $\mathrm{TC} \dagger$ & A-I & A-II & $\mathrm{B}$ & C-I & C-II & C-III & E \\
\hline $\begin{array}{l}\text { Before GDF } \\
\quad(-1-0 y)\end{array}$ & $434 \pm 343$ & $195 \pm 84$ & $104 \pm 27$ & $64 \pm 22$ & $135 \pm 48$ & $11.6 \pm 5.7$ & $5.7 \pm 1.4$ & $18.6 \pm 9.9$ & $17.2 \pm 14$ \\
\hline $\begin{array}{l}\text { After GDF }(0-1 \\
\text { y) }\end{array}$ & $568 \pm 372$ & $227 \pm 61$ & $96 \pm 27$ & $57 \pm 18$ & $155 \pm 45$ & $9.0 \pm 3.1$ & $6.0 \pm 1.8$ & $19.3 \pm 9.0$ & $18.9 \pm 7.7$ \\
\hline $\begin{array}{l}\text { After GDF (1-2 } \\
\text { y) }\end{array}$ & $400 \pm 410$ & $185 \pm 79$ & $103 \pm 27$ & $60 \pm 16$ & $130 \pm 63$ & $6.2+$ & $5.0 \div$ & $19.0 \pm 17.5$ & $14.8 \pm 6.9$ \\
\hline $\begin{array}{l}\text { After GDF (2-5 } \\
\text { y) }\end{array}$ & $398 \pm 314$ & $201 \pm 73$ & $102 \pm 34$ & $53 \pm 11$ & $109 \pm 47$ & $11.2 \pm 1.2$ & $6.1 \pm 1.6$ & $20.7 \pm 11.9$ & $17.8 \pm 6.5$ \\
\hline $\begin{array}{l}\text { After GDF (5-8 } \\
\text { y) }\end{array}$ & $601 \pm 557$ & $246 \pm 101$ & $107 \pm 25$ & $57 \pm 10$ & $158 \pm 79$ & $12.0 \pm 6.7$ & $7.7 \pm 2.5$ & $27.6 \pm 18$ & $21.6 \pm 10.7$ \\
\hline Control subjects & $92 \pm 40$ & $181 \pm 30$ & $104 \pm 22$ & $15 \pm 11$ & $92 \pm 21$ & $9.0 \pm 2.6$ & $3.2 \pm 1.2$ & $7.2 \pm 1.6$ & $8.5 \pm 3.2$ \\
\hline
\end{tabular}

* TG, triglycerides.

+ TC. total cholesterol.

$\ddagger$ Single measurement.

Table 3. Plasma lipid and apolipoprotein concentrations (in $\mathrm{mg} / \mathrm{dL}$ ) (mean \pm SD) in patients with GSD-IA and GSD-IB, not responding to GDF treatment (cases 15-20) and in control subjects (Ref. 6)

\begin{tabular}{|c|c|c|c|c|c|c|c|c|c|}
\hline \multirow[b]{2}{*}{ Treatment } & \multicolumn{2}{|c|}{ Lipids } & \multicolumn{7}{|c|}{ Apolipoproteins } \\
\hline & $\mathrm{TG}^{*}$ & TCt & A-I & A-II & B & C-I & C-II & C-III & E \\
\hline Before GDF $(-1-0$ y) & $479 \pm 389$ & $143 \pm 59$ & $113 \pm 43$ & $59 \pm 17$ & $137 \pm 58$ & $15.4 t$ & $5.0 \ddagger$ & $23.0 \pm 11$ & $16.2 \pm 8.0$ \\
\hline After GDF $(0-1$ y) & $447 \pm 133$ & $155 \pm 35$ & $82 \pm 19$ & $41 \pm 8.7$ & $121 \pm 24$ & $13.0 \ddagger$ & $5.0 \ddagger$ & $25.7 \pm 9.0$ & $12.6 \pm 8.2$ \\
\hline After GDF $(1-2 y)$ & $485 \pm 297$ & $218 \pm 36$ & $87 \pm 28$ & $54 \pm 22$ & $162 \pm 41$ & $10.6 \pm 2.1$ & $4.6 \ddagger$ & $26.5 \pm 9.3$ & $21.1 \pm 18.5$ \\
\hline After GDF $(2-5 y)$ & $692 \pm 314$ & $236 \pm 58$ & $77 \pm 31$ & $51 \pm 17$ & $155 \pm 67$ & $9.4 \pm 3.3$ & $11.6+$ & $21.5 \pm 6.7$ & $17.7 \pm 9.7$ \\
\hline After GDF (5-8 y) & $1625 \pm 1360$ & $\begin{array}{l}390 \pm 163 \\
p<0.05\end{array}$ & $71 \pm 22$ & $45 \pm 6.3$ & $260 \pm 72$ & $17.2 \pm 15$ & $8.4 \pm 1.8$ & $33.8 \pm 11.2$ & $39.0 \pm 27$ \\
\hline Control & $92 \pm 40$ & $181 \pm 30$ & $104 \pm 22$ & $55 \pm 11$ & $92 \pm 21$ & $9.0 \pm 2.6$ & $3.2 \pm 1.2$ & $7.2 \pm 1.6$ & $8.5 \pm 3.2$ \\
\hline
\end{tabular}

* TG, triglycerides.

† TC. total cholesterol.

S Single measurement.

\$ Significantly different from the mean cholesterol value for patients before GDF. 
proteins of both responders and nonresponders were significantly different from those of normolipidemic, asymptomatic children (6).

\section{DISCUSSION}

Improvement of growth in GSD-I patients, due to treatment with nocturnal GDF in conjunction with frequent feedings during the day, has been reported by many authors $(1-3,13)$. The favorable effects of GDF have been attributed to the constant provision of fuel to peripheral tissues (14). This is associated with a decrease of plasma glucagon and an increase of plasma insulin and growth hormone $(1,13)$. These hormonal changes as well as an amelioration of the tendency toward lactic acidosis (3) and a normalization of the sleep pattern (2) may be factors contributing to the improvement of growth.

We used two criteria to substantiate a favorable response of the patients' growth to long-term GDF treatment. First, a significant upward deviation of height SDS from the track of growth before the institution of GDF. The calculation of significance was obtained by comparing the $\triangle \mathrm{SDS}$ over 1-, 2-, and 5-y periods with the $95 \%$ confidence intervals of $\triangle$ SDS of a healthy Finnish child population (10). This is applicable to growth-retarded patients showing catch-up growth, but not to patients who maintained a normal track before and during GDF. We defined the latter patients also as responders to GDF as long as their SDS remained above -2 and $\Delta$ SDS did not show a significant downward deviation. Using these two criteria, we consider our patients $1-11$ to be definite responders, and cases $12-14$ as probable responders.

Unlike these 14 responders, the other six patients reacted less favorably to GDF. Two patients (cases 15 and 16) showed only a temporary catch-up growth followed by deceleration. Three patients (cases 18-20) had a clearly downward deviation of height SDS.

Such a division of patients into responders and nonresponders might suggest the existence of two distinct groups of patients. However, this is obviously not the case because a transition zone exists between patients who respond conspicuously favorably to GDF and those who did improve with respect to vitality, appetite, excessive sweating, and sleep pattern but not with respect to growth. Yet, having defined some patients as nonresponders, we increased our efforts to search for dietary imperfections, endocrinologic abnormalities, and environmental interferences, but we failed to find any explanation for their insufficient growth during GDF. Thus, the decelerated growth could not be due to advanced glomerular sclerosis (15), because of 14 patients in whom glomerular filtration rate and renal plasma flow were determined, increased values were found in nine and 12 patients, respectively; none of the patients showed decreased values. Tubular function, as estimated by the urinary excretion of $\beta_{2}$ microglobulin and lysozyme, was normal. The abnormalities of renal function were not related to the growth rate of the patient, to the age at which GDF had been instituted, or to the age of the patient when tested.

With respect to the abnormalities of plasma lipids and apolipoproteins, which closely resembled those of Levy et al. (16), no statistically significant differences existed between responders and nonresponders before the initiation of GDF treatment. GDF had a marginal effect on plasma lipids and apolipoprotein profiles after $1-2 \mathrm{y}$, but only in those patients whose growth responded favorably. These effects wore off after continued treatment, and ultimately plasma triglycerides, cholesterol, apolipoproteins B, C-III, and E increased to higher concentrations, and, conversely, apolipoproteins A-I and A-II decreased to lower concentrations than those characteristic of the pretreatment period, particularly in nonresponders. Therefore, neither the pre-GDF profile of plasma lipids and apolipoproteins, nor the post-GDF changes of the profile allowed one to predict if a patient's growth would respond favorably to GDF treatment, whereas a good correlation appears to exist between growth and such other metabolic abnormalities as 2-oxoglutarate and lactate (4).

The atherogenic character of the apolipoprotein profile (17) was not reflected in clinical symptoms of premature atherosclerosis in our patients. In four patients, aged 10,14,15, and $16 \mathrm{y}$, ultrasound study of the heart and ECG during excercise did not reveal abnormalities.

Probably the increased tendency to atherosclerosis that might be expected from the apolipoprotein profile is offset by the decreased adhesiveness of the platelets, characteristic of GSD I (18). This would tally with the resistence to atherosclerosis in pigs with Von Willebrand's disease (19) and with the antiatherogenic effect of such platelet-inhibiting drugs as aspirin in the human.

The absence of atherosclerotic symptoms in our patients does not mean that the abnormalities of plasma lipids and apolipoproteins do not have pathologic implications. Although no etiologic mechanism is known, the elevated lipid and apolipoprotein levels may be involved in the development of glomerular disease (20), the initial phase of which was found in 10 of our 20 patients. We have previously suggested (6) that the molar ratio of apolipoproteins $\mathrm{B} / \mathrm{C}-\mathrm{III} / \mathrm{E}$ of $1: 4-5: 1$ might reflect mainly the overproduction of some triglyceride-rich lipoprotein particles (21). The increased liponeogenesis may be the consequence of an enhanced glycogenolytic flux through an enlarged glucose-6phosphate pool (22), resulting not only in increased pyruvate and lactate formation (4) but in increased production of malonyl$\mathrm{CoA}$ as well. The latter is the main substrate of fatty acid synthesis from pyruvate (23). Thus, lactate overproduction, which has been shown to be very sensitive to insufficient glucose availability (4), may be the first outlet of excessive glycogenolysis; endogenous triglyceride overproduction reflected in hyperlipoproteinemia would be a relatively insensitive and quantitatively larger second outlet (3). This abnormality appears to be less easily corrected by GDF than growth, the latter being usually, though not always, responsive to dietary manipulation.

\section{REFERENCES}

1. Greene HL, Slonim AE, O’Neill JA, Burr IM 1976 Continuous nocturnal intragastric feeding for management of type I glycogen storage disease. $\mathrm{N}$ Engl J Med 294:423-425

2. Fernandes J, Jansen $H$, Jansen TC 1979 Nocturnal gastric drip feeding in glucose-6-phosphatase deficient children. Pediatr Res 13:225-229

3. Stanley CA, Mills JL, Baker L 1981 Intragastric feeding in type I glycogen storage disease: factors affecting the control of lactic acidemia. Pediatr Res 15:1504-1508

4. Fernandes J, Berger R 1987 Urinary excretion of lactate, 2-oxoglutarate, citrate and glycerol in patients with glycogenosis type I. Pediatr Res 21:279-282

5. Rosenfeld EL, Chibisov IV, Karmansky IM, Tabolin VA, Chistova LV, Leontiev AF 1980 Serum lipoproteins of patients with glycogen storage disease. Clin Chim Acta 102:99-104

6. Alaupovic P, Fernandes J 1985 The serum apolipoprotein profile of patients with glucose-6-phosphatase deficiency. Pediatr Res 19:380-384

7. Tanner JM, Whitehouse RH, Hughes PCR, Vince FP 1971 Effects of human growth hormone treatment for 1 to 7 years on growth of 100 children, with growth hormone deficiency, low birthweight, inherited smallness, Turner's syndrome and other complaints. Arch Dis Child 46:745-782

8. Roede MJ, Van Wieringen JC 1985 Growth diagrams 1980. Netherlands third nation-wide survey. Tijdschr Soc Gezondheidszorg 63 (Suppl): 1-34

9. Van Wieringen JC, Wafelbakker F, Verbrugge HP, De Haas JH 1971 Growth Diagrams 1965 The Netherlands. Wolters-Noordhoff, Groningen

10. Sorva R, Tolppanen EM, Lankinen S, Perheentupa J 1985 Evaluation of childhood growth. A review (in Finnish). Duodecim 101:465-476

11. Huang TC, Chen CP, Wefler V, Raferty A 1961 A stable reagent for the Lieberman-Burchard reaction: application to rapid serum cholesterol determination. Anal Chem 33:1405-1407

12. Soloni FG 1971 Simplified manual micromethod for determination of serum triglyceride. Clin Chem 17:529-534

13. Greene HL, Slonim AE, Burr IM, Moran JR 1980 Type I glycogen storage disease: five years of management with nocturnal intragastric feeding. $J$ Pediatr 96:590-595

14. Williams JC 1986 Nutritional goals in glycogen storage disease. N Engl J Med 314:709-710

15. Chen YT, Coleman RA, Scheinman JI, Kolbeck PC, Sidbury JB 1988 Renal disease in type I glycogen storage disease. N Engl J Med 318:7-11

16. Levy E, Thibault LA, Roy CC, Bendayan M, Lepage G, Letarte J 1988 Circulating lipids and lipoproteins in glycogen storage disease type I with nocturnal intragastric feeding. J Lipid Res 29:215-226 
17. Thompson G 1984 Apoproteins: determinants of lipoprotein metabolism and indices of coronary risk. Br Heart J 51:585-588

18. Corby DG, Putman CW, Greene HL 1974 Impaired platelet function in glucose-6-phosphatase deficiency. J Pediatr 85:71-76

19. Fuster V, Bowie EJW, Lcwis JC, Fass DN 1978 Resistance to arteriosclerosis in pigs with Von Willebrand's disease. J Clin lnvest 61:722-730

20. Moorhead JF, Chan MK, El-Nahas M, Varghese Z 1982 Lipid nephrotoxicity in chronic progressive glomerular and tubulo-interstitial disease. Lancet 2:1309-1311
21. Chait A, Albers JJ, Brunsell JD 1980 Very low density lipoprotein overproduction in genetic forms of hypertriglyceridemia. Eur J Clin Invest 10:1722

22. Oberhaensli RD, Rajagopolan B, Taylor DJ, Radda GK, Collins JE, Lconard JV 1988 Study of liver metabolism in glucose-6-phosphatase deficiency by P-31 magnetic resonance spectroscopy. Pediatr Res 23:375-380

23. McGarry JD, Mannaerts GP, Foster DW 1977 A possible role for malonyl$\mathrm{COA}$ in the regulation of hepatic fatty acid oxidation and ketogenesis. $\mathrm{J}$ Clin Invest 60:265-270 